\title{
Edema óseo secundario a lesiones ligamentarias de tobillo. Hallazgos en las imágenes de resonancia magnética
}

\author{
Manel Ballester-Alomar," Gustavo Lucar-López," Federico Bugallo," Griselda March-Sorribes," \\ Praneeth Revuri,"\# Kerbi A. Guevara Noriega ${ }^{ \pm}$ \\ *Unidad Pie y Tobillo, Servicio de Ortopedia y Traumatología, Hospital de Mataró, Barcelona, España \\ ** Equipo Miembro Inferior, División Ortopedia y Traumatología, Hospital General de Agudos "Dr. Juan A. Fernández", \\ Ciudad Autónoma de Buenos Aires, Argentina \\ "Unidad Podología, Clínica Creu Blanca, Barcelona, España \\ \#Cirugía de Tobillo, Mlathi Manipal Hospitals, Jayanagar, Bangalore, India \\ ${ }^{\ddagger}$ General Surgery, Jackson Memorial Hospital, Miami, Estados Unidos
}

\section{RESUMEN}

Introducción: El esguince agudo de tobillo es una de las lesiones musculoesqueléticas más frecuentes. Su creciente estudio con resonancia magnética ha llevado a una mayor detección de edema óseo. El edema óseo por contusión consiste en una fractura ósea subcondral microtrabecular del hueso esponjoso junto con hemorragia local y edema. El objetivo de este estudio fue analizar una posible relación estadística entre la lesión ligamentaria de tobillo y la localización del edema óseo. Materiales y Métodos: Entre 2016 y 2018, se analizaron 50 resonancias de pacientes con diagnóstico clínico de esguince sin lesión ósea en la radiografía. Dos radiólogos independientes analizaron las secuencias T1 y T2 en los planos coronal, axial y sagital. Se comparó la localización del edema óseo y las lesiones ligamentarias con el fin de encontrar posibles asociaciones. Resultados: No hubo diferencias en la presencia de edema óseo en relación con el ligamento lesionado como consecuencia de un esguince de tobillo $(p>0,05)$. En general, el edema óseo fue más frecuente en la cabeza y el cuello del astrágalo (64\%). No se halló una relación estadística entre la localización de edema óseo y el ligamento lesionado $(p>0,05)$. Conclusiones: El edema óseo es un hallazgo frecuente en la resonancia magnética después de una lesión ligamentaria a causa de esguinces de tobillo; sin embargo, no hay evidencia de una relación entre la localización del edema y el ligamento lesionado.

Palabras clave: Resonancia magnética; esguince; tobillo; edema óseo.

Nivel de Evidencia: II

\section{Bone Marrow Edema Following Ankle Ligament Injuries: an MRI Imaging Evaluation}

\begin{abstract}
Acute ankle sprains are a common type of musculoskeletal injury. With the increased popularity of MRI post-ankle sprain, more cases of bone marrow edema or bone bruise are detected. This is a subchondral osseous fracture of the cancellous microarchitecture with accompanied local hemorrhage and edema. This study aims to analyze if the presence of ankle ligament injury presents any significant statistical relation with the specific location. Materials and Methods: We reviewed 50 MRI performed during 20152018 for patients who reported ankle pain and signs of instability. Two different radiologists evaluated the series. Bone marrow edema location and specific ligament lesions were contrasted to find possible associations. Chi-Square was used to analyze the differences. Results: The anterior talofibular ligament was the most commonly injured ligament (84\%). CFL injury occurred in 27 cases $(54 \%)$. There were no differences between the presence of bone marrow edema and the ligament injured after an ankle sprain ( $p>0.05$ ). Overall, the most common location of bone marrow edema was the head and neck of the talus (64\%). There was no statistical relationship between bone marrow edema location and the injured ligament $(p>0.05)$. Conclusion: Bone marrow edema is a frequent finding after a ligament injury following ankle sprains. However, this finding is not directly related to a specific location of the injured ligament. The location of bone marrow edema is not related to specific ligament injuries. MRI findings of bone marrow edema must not change the management of patients with acute ankle sprains.
\end{abstract}

Key words: MRI; ankle sprain; bone marrow.

Level of Evidence: II

Recibido el 21-5-2020. Aceptado luego de la evaluación el 20-7-2020 • Dr. MANEL BALLESTER-ALOMAR • manel.ballester@gmail.com

https://orcid.org/0000-0001-5351-605X

Cómo citar este artículo: Ballester-Alomar M, Lucar-López G, Bugallo F, March-Sorribes G, Revuri P, Guevara Noriega KA. Edema óseo secundario a lesiones ligamentarias de tobillo. Hallazgos en las imágenes de resonancia magnética. Rev Asoc Argent Ortop Traumatol 2021;86(1):17-22. https://doi.org/10.15417/issn.1852-7434.2021.86.1.1124 


\section{INTRODUCCIÓN}

El esguince de tobillo representa un cuadro muy frecuente en la práctica diaria de la traumatología, tanto en la emergencia como en una consulta externa. Diferentes estudios en los Estados Unidos y Europa han comprobado que el 30\% de las lesiones en los atletas implican esguinces de tobillo. La clínica descrita más frecuente es dolor en la parte lateral del tobillo e inestabilidad luego de una flexión plantar e inversión. ${ }^{1} \mathrm{Al}$ hablar de esguince de tobillo, hay consenso en que nos referimos a la lesión de las estructuras ligamentarias que forman parte de dicha articulación. Puede ser desde una contusión, con lo cual los signos y síntomas pueden limitarse simplemente a dolor o edema regional, o bien puede tratarse de una rotura total de uno o más componentes que causa una sintomatología más florida en la etapa aguda.

El complejo ligamentario lateral del tobillo está compuesto por el ligamento peroneo-astragalino anterior (LPAA), el ligamento peroneo-calcáneo (LPC) y el ligamento peroneo-astragalino posterior (LPAP). Por lo general, hay un ángulo de $120^{\circ}$ entre el LPAA y el LPC. En un esguince de tobillo agudo, el LPAA resulta ser el más afectado, seguido del LPC. El LPAP es el más robusto y, por lo tanto, el que menos posibilidades de rotura tiene. Además, este se ve favorecido por la cinemática del trauma, la cual se dirige de anterior a posterior, por lo que la lesión aislada de este ligamento es infrecuente en los esguinces.

Las revisiones sistemáticas han demostrado la persistencia del dolor y la inestabilidad en el 5-33\% de los pacientes después de un tratamiento conservador. El $34 \%$ de los pacientes refirió, al menos, un esguince recurrente y el $15-64 \%$, solo una recuperación parcial de la lesión inicial. ${ }^{2}$

Asimismo, resulta fundamental observar las lesiones que pueden presentarse asociadas con el esguince lateral de tobillo, tales como desgarros del tendón peroneo, lesiones osteocondrales o cuerpos libres intrarticulares, que pueden provocar signos crónicos si no se tratan de forma apropiada. A pesar de que se puede detectar un defecto osteocondral oculto en una resonancia magnética (RM), su tamaño y extensión pueden sobrestimarse debido a la cantidad de edema óseo alrededor de la lesión. Por esta razón, este estudio se debe complementar con una tomografía computadorizada (TC). Estas lesiones pueden identificarse en la RM, pero este estudio representa un recurso menos disponible debido a su acceso o costo, o a razones administrativas, por ejemplo, en ocasiones, los seguros médicos no la cubren.

Se sabe que la mayoría de estos pacientes obtiene buenos resultados con protocolos de terapias físicas fuertes implementados correctamente con entrenamiento propioceptivo agresivo. Uno de los problemas que se deben considerar es que la inmovilización durante 4-6 semanas puede provocar la rigidez de la articulación del tobillo y así agravar aún más la situación.

Frey y cols. han demostrado que un adecuado examen físico puede diagnosticar las lesiones grado III con un $100 \%$ de precisión. Dicho porcentaje es ostensiblemente más bajo en las lesiones grados I y II. ${ }^{3}$ A esta dificultad se suma que se diagnostican menos los cuadros asociados.

Por este motivo, las radiografías con estrés en varo-valgo y cajón anterior en $20^{\circ}$ y $0^{\circ}$ de flexión plantar son de vital importancia para evaluar la funcionalidad del LPAA y el LPC, respectivamente.

En algunos casos, puede haber una atenuación del ligamento deltoideo. Resulta muy importante también controlar la prueba de inclinación talar (talar tilt test) durante el examen físico, pese a que sea menos frecuente en pacientes con esguinces laterales de tobillo.

Ramsey y Hamilton han demostrado que, con un desplazamiento lateral de $2 \mathrm{~mm}$, el contacto tibio-astragalino disminuye un $42 \%$. Esto puede explicar la razón de la artritis postraumática y la importancia de obtener un diagnóstico rápido para evitar posibles complicaciones. Cuando el examen físico no resulta suficiente para diagnosticar un esguince lateral agudo de tobillo, la opción más frecuente es la RM. La exactitud para el diagnóstico de un desgarro del LPAA fue del 91,7\%; para un desgarro del LPC, del 87,5\% y para lesiones osteocondrales del 83,3\%. La sensibilidad fue baja, sobre todo en los desgarros del LPC; por este motivo, la cantidad de falsos negativos es alta. ${ }^{4,5}$

Un análisis de la bibliografía revela una discusión controvertida sobre el hallazgo del edema óseo en la RM observado en los sitios de inserción de los ligamentos laterales del tobillo (LPAA, LPC, LPAP), y su papel como indicador diagnóstico adicional para iniciar un tratamiento apropiado de esguinces agudos de tobillo (Figura 1). Asimismo, se debe prestar atención a los falsos negativos en las RM; sin embargo, si se presenta un edema óseo, esto debe considerarse como una bandera roja que indica atenuación del LPAA y el LPC. ${ }^{6}$

Por este motivo, es prioritario conocer si existe una correlación clínica/radiológica y, por ende, dirigir, en un sentido u otro, el manejo terapéutico. El objetivo de este estudio fue evaluar la relación entre la presencia de edema óseo y las lesiones ligamentarias específicas luego de un esguince de tobillo. Se busca analizar la relación entre el edema óseo identificado por la RM y las lesiones ligamentarias de tobillo en localizaciones específicas. 


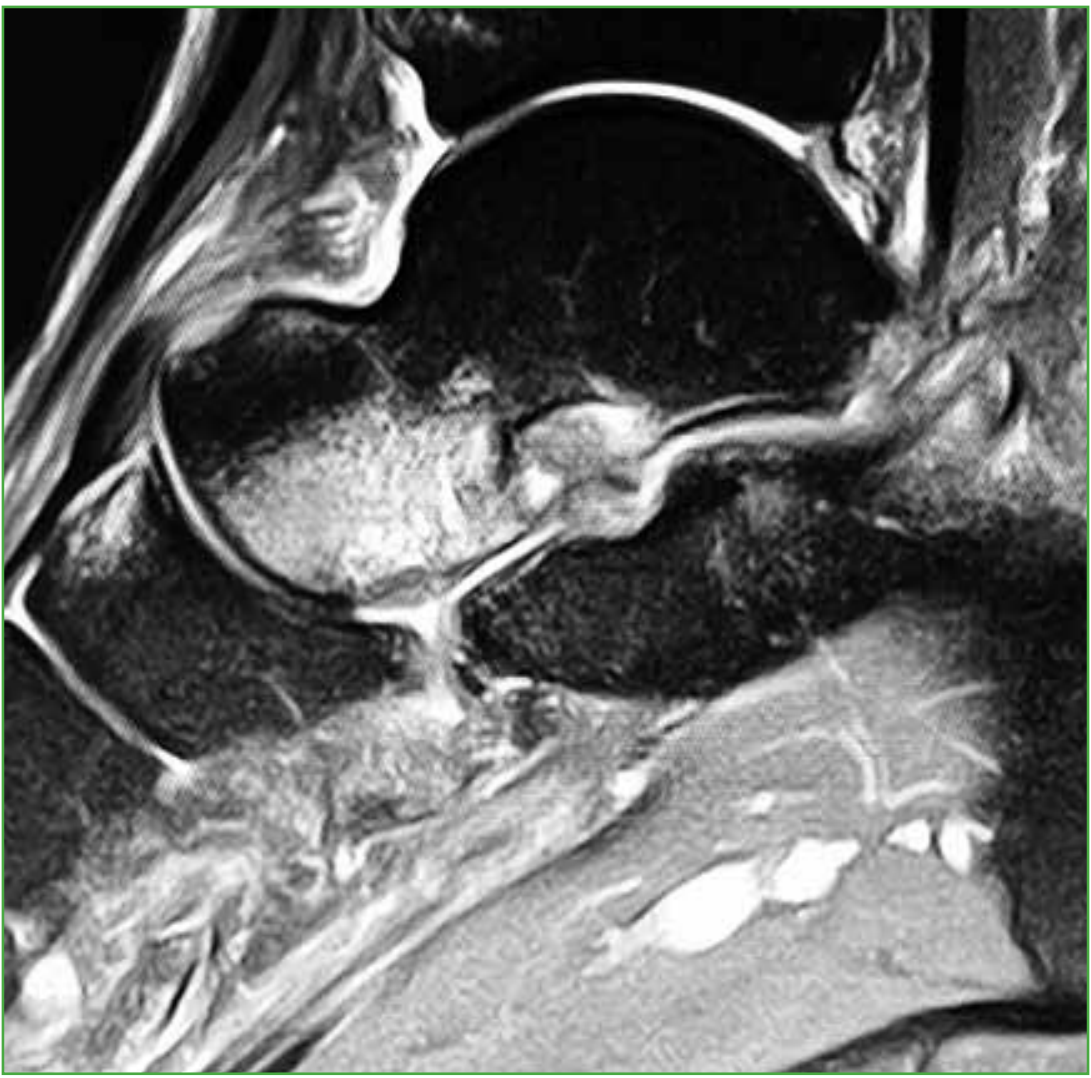

Figura 1. Edema óseo poscontusivo en el cuello y la cabeza del astrágalo.

\section{MATERIALES Y MÉTODOS}

Se analizaron 50 RM realizadas entre 2016 y 2018 a pacientes con diagnóstico clínico de esguince de tobillo, con dolor o signos de inestabilidad, sin lesión ósea en las radiografías. El promedio de edad era de $24 \pm 10.7$ años (rango 15-64). Diez pacientes eran mujeres y 20, hombres.

Las RM se realizaron, en promedio, $23 \pm 39.8$ días después del trauma (rango 3-210). Dos radiólogos independientes evaluaron secuencias T1 y T2, en los planos coronal, axial y sagital en busca de las características del: 1) LPAA, 2) LPC, 3) LPAP y 4) ligamento deltoideo (LD). También se evaluó la presencia de edema óseo y su ubicación se definió de acuerdo con las siguientes localizaciones: a) domo astragalino medial, b) domo astragalino lateral, c) cuerpo del astrágalo, d) cuello y cabeza del astrágalo, e) maléolo medial, f) maléolo lateral, g) maléolo posterior, h) calcáneo. Con estos datos, se procedió a realizar un análisis de estos parámetros.

Se excluyó a los pacientes con antecedentes de lesiones ligamentarias u osteocondrales y a aquellos con inestabilidad ligamentaria crónica.

Se utilizó el programa SPSS 20 para el análisis estadístico. Se analizó una relación estadística significativa entre el edema óseo y una localización específica de la lesión ligamentaria. Se aplicó la prueba de ji al cuadrado.

\section{RESULTADOS}

Según las 50 RM analizadas, el LPAA fue el más afectado por lesiones (42 casos, 84\%). En el 55\%, la lesión era total y, en el 26\%, parcial. La lesión en el LPAA se asoció, con frecuencia, a una lesión simultánea de otro ligamento; solo, en cinco casos, la lesión era aislada y, en tres de ellos (60\%), el edema estaba en la cabeza y el cuello del astrágalo sin un patrón claramente definido.

Se observó una lesión del LPC en 27 casos (54\%); en el 63\%, era parcial y, en el 37\%, total. No se detectaron casos de lesión aislada del LPC. En 14 de los casos (52\%), la lesión del LPC estaba relacionada con la del LD. Cuando la lesión era doble (LPC más LPAA), el edema óseo apareció en el cuerpo del astrágalo (12 pacientes, 
$24 \%$ ) y en la cabeza y el cuello (9 pacientes, 18\%). Sin embargo, hubo ocho casos de lesión ligamentaria triple (LPC más LPAA más LD); el edema óseo se detectó, con mucha frecuencia, en el cuerpo del astrágalo, su cabeza o cuello, pero también en el maléolo medial (4 casos) y lateral ( 2 casos).

Solo un paciente tenía una lesión total del complejo ligamentario lateral (LPAA más LPC más LPAP más LD). En este escenario, el edema óseo se detectó en la cabeza del astrágalo y en el maléolo medial.

Veinticinco pacientes (50\%) tenían lesión del LD. Hubo solo un caso de lesión total. Se detectaron 24 lesiones parciales: 18 ocurrieron junto con las lesiones del LPAA y el LPC y, en los seis casos restantes, solo junto con la del LPAA.

La localización más frecuente del edema óseo fue la cabeza y el cuello del astrágalo (32 pacientes, 64\%), comúnmente cuando la RM mostró una lesión lateral o medial (15 casos, 30\%). La segunda localización más común fue el cuerpo del astrágalo (20 pacientes, $40 \%$ ) (Figura 2).

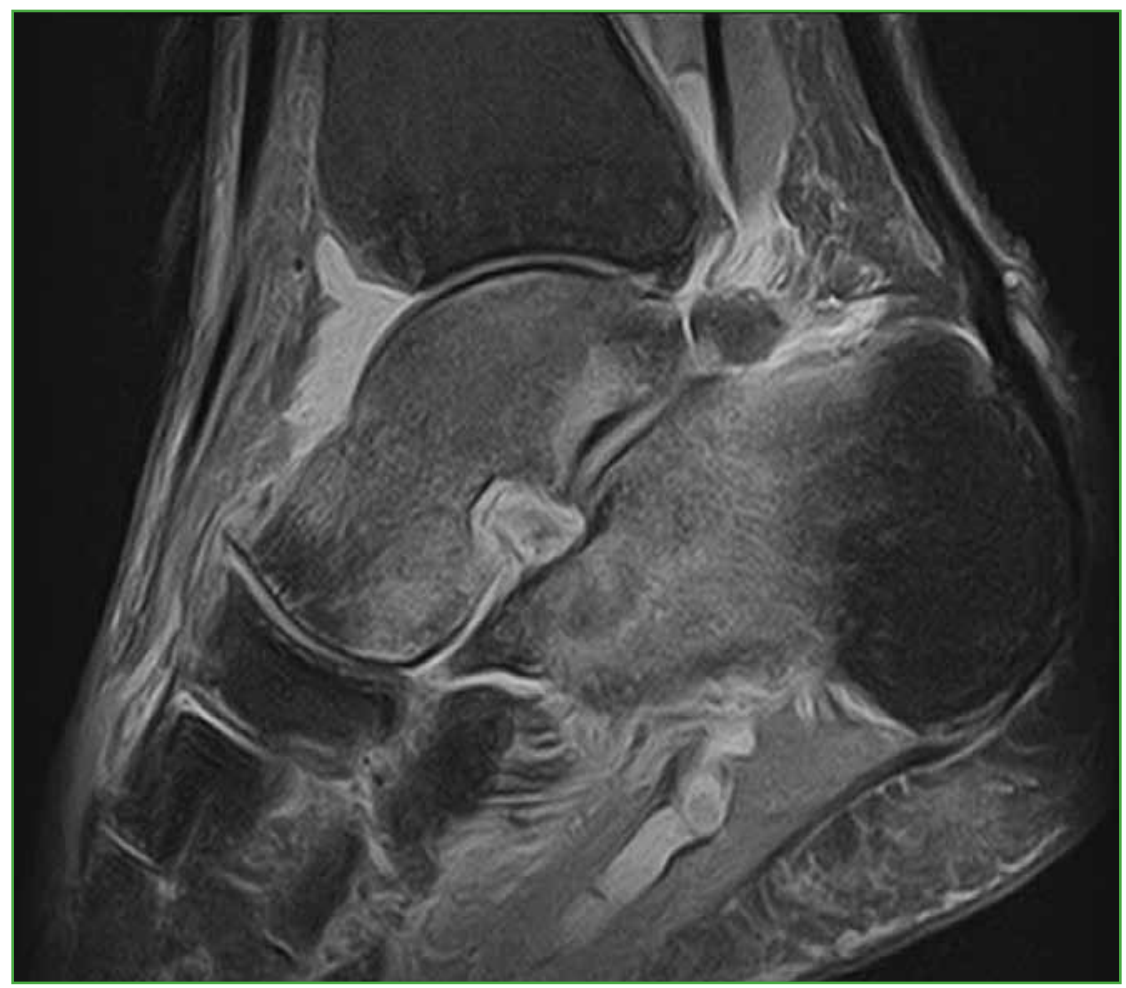

Figura 2. Edema óseo poscontusivo en el cuerpo del astrágalo y el calcáneo.

El 30\% y el $40 \%$ de los casos tenían edema en el maléolo, ya sea medial o lateral, respectivamente, y resultó aún más común cuando dos o más ligamentos estaban lesionados.

Se detectó edema de domo astragalino en 15 casos (30\%), fue más frecuente en la zona medial (13 casos, 26\%), y edema de domo astragalino lateral en cuatro casos. El edema de domo astragalino no tuvo un patrón de desarrollo claramente definido acorde con el tipo de lesión.

No se detectó ninguna relación estadística entre la localización del edema óseo y el ligamento lesionado ( $\mathrm{p}>0,05)$.

\section{DISCUSIÓN}

Este estudio incluyó principalmente a pacientes que sufrieron esguinces de tobillo como consecuencia de la práctica de deportes de contacto, sobre todo, básquetbol. El mecanismo de lesión observado más común es la combinación de flexión plantar e inversión, aunque también se detectan otras combinaciones, que provocan lesiones combinadas, tanto del complejo medial como de la sindesmosis y la articulación subastragalina. La lesión parcial 
suele ser más común y, en todos los casos, asociada a edema óseo por contusión. Es importante señalar que, de acuerdo con lo esperado, nuestro estudio detectó más casos de edema óseo por RM que mediante el uso aislado de radiografía. Más relevante aún fue el hallazgo de casos de edema óseo sin lesión ligamentaria. Todo ello lleva a concluir que la RM es una técnica más sensible y permite detectar edema óseo si no hay una lesión ligamentaria, algo que resulta interesante y que puede justificar el dolor aun si no hay una fractura o un esguince.

Es importante destacar que existen diferentes maneras de visualizar alteraciones en la RM. Las lesiones óseas se observan como hipointensidad en la secuencia T1, mientras que el edema o la hemorragia se detectan como imágenes isointensas. En la secuencia T2, las lesiones óseas se visualizan como zonas de heterogeneidad de intensidad de la señal y el edema o las hemorragias, como imágenes hiperintensas. Esto hace posible confirmar la presencia de un edema óseo sin mucha dificultad y su posible asociación a fracturas. ${ }^{7}$

En la RM, la aparición de lesiones lineales y geográficas sugiere una lesión más localizada, incluso puede estar comprometido también el cartílago subyacente. La lesión oculta del cartílago puede no ser detectada en la RM. Esto podría ser una justificación del dolor del paciente si no hay lesión ligamentaria o fractura, y se necesitan más estudios para aclarar este punto en particular.

Mink y Deutsch dividen las lesiones óseas ocultas en cuatro categorías: edema óseo por contusión, fractura por fatiga, fracturas femorales y tibiales, y fracturas osteocondrales. ${ }^{8}$ En futuras investigaciones, resultaría útil asociar esta clasificación con variables clínicas.

Para nuestro objetivo en particular, cuando analizamos la relación entre el patrón de las lesiones ligamentarias y los edemas óseos por contusión, no se halló un patrón claro o una específica constante en la distribución del edema óseo en los esguinces de tobillo. Los resultados confirman las conclusiones de Labovitz y cols. quienes publicaron resultados similares y concluyeron en que no es sencillo establecer una relación entre las lesiones ligamentarias de tobillo y la presencia de edema óseo por contusión. Asimismo, el edema óseo por contusión sin lesiones ligamentarias o fracturas lleva a pensar que se trata de un signo radiológico poco específico. ${ }^{9}$

La bibliografía apoya el manejo conservador como elección de primera línea para los esguinces de tobillo grados I y II. Si se tienen en cuenta nuestros resultados, este manejo sigue en pie y no tiene ninguna utilidad detectar edema óseo en la RM, en este tipo de lesiones.

Este tratamiento se asocia con buenos resultados; sin embargo, si no se realiza correctamente, pueden aparecer secuelas a largo plazo. El método RICE (Rest, Ice, Compression, Elevation [Reposo, Hielo, Compresión, Elevación]) parece suficiente hasta que la inflamación y el edema de los tejidos blandos disminuyan, seguido de deambulación con apoyo parcial o total del peso corporal, según la tolerancia al dolor. En este contexto, sería recomendable asociar la rehabilitación con un fisioterapeuta para disminuir la posibilidad de secuelas. ${ }^{10}$

La causa más frecuente de las lesiones es la inversión forzada durante la práctica de deportes, caídas o accidentes de tránsito. Además, la eversión y abducción del pie suelen estar asociadas a otra lesión de otro ligamento o tendón, y los edemas óseos por contusión se han descrito como signos indirectos de la lesión ligamentaria., ${ }^{411-14}$

Este estudio tiene ciertas limitaciones, pues se trata de un análisis retrospectivo que solo se centró en los edemas óseos por contusión y lesiones ligamentarias, es decir, rotatoria, varo, impacto valgo o combinados. El seguimiento fue adecuado y superior al 90\%. A algunos pacientes se les tomó una segunda RM con el fin de analizar los edemas óseos por contusión. Como se evaluó a una cantidad limitada de pacientes y el análisis primario no demostró ninguna utilidad, no resulta correcto o útil extraer conclusiones sobre este segundo estudio.

\section{CONCLUSIONES}

El edema óseo es común luego de una lesión ligamentaria tras un esguince de tobillo. Sin embargo, la localización anatómica del edema óseo no está directamente relacionada con una localización específica del ligamento lesionado. El edema óseo detectado en una RM no debe implicar un cambio en el tratamiento de los pacientes con esguince agudo de tobillo. Aun así, la RM sigue siendo una herramienta de suma utilidad para detectar lesiones asociadas.

Conflicto de intereses: Los autores no declaran conflictos de intereses. 
ORCID de G. Lucar-López: https://orcid.org/0000-0002-7882-6289 ORCID de F. Bugallo: https://orcid.org/0000-0002-7285-967X

ORCID de G. March-Sorribes: https://orcid.org/0000-0001-9980-9800
ORCID de P. Revuri: https://orcid.org/0000-0002-6036-9411

ORCID de K. A. Guevara Noriega: https://orcid.org/0000-0003-2643-6390

\section{BIBLIOGRAFÍA}

1. Saxena A, Luhadiya A, Ewen B, Goumas C. Magnetic resonance imaging and incidental findings of lateral ankle pathologic features with asymptomatic ankles. J Foot Ankle Surg 2011;50:413-5.

https://doi.org/10.1053/j.jfas.2011.03.011

2. Van Rijn RM, van Os AG, Bernsen RM, Luijsterburg PA, Koes BW, Bierma-Zeinstra SM. What is the clinical course of acute ankle sprains? A systematic literature review. Am J Med 2008;121:324-31. https://doi.org/10.1016/j.amjmed.2007.11.018

3. Frey C, Bell J, Teresi L, Kerr R, Feder K. A comparison of MRI and clinical examination of acute lateral ankle sprains. Foot Ankle Int 1996;17:533-7. https://doi.org/10.1177/107110079601700904

4. Park H-J, Cha S-D, Kim SS, Rho M-H, Kwag H-J, Park N-H, Lee S-Y. Accuracy of MRI findings in chronic lateral ankle ligament injury: comparison with surgical findings. Clin Radiol 2012;67:313-8. https://doi.org/10.1016/j.crad.2011.08.025

5. Ramsey PL, Hamilton W. Changes in tibiotalar area of contact caused by lateral talar shift. J Bone Joint Surg Am 1976;58:356-7. PMID: 1262367

6. van Putte-Katier N, van Ochten JM, van Middelkoop M, Bierma-Zeinstra SM, Oei EH. Magnetic resonance imaging abnormalities after lateral ankle trauma in injured and contralateral ankles. Eur J Radiol 2015;84:2586-92. https://doi.org/10.1016/j.ejrad.2015.09.028

7. Lee JK, Yao L. Occult intraosseous fracture: magnetic resonance appearance versus age of injury. Am J Sports Med 1989;17:620-3. https://doi.org/10.1177/036354658901700505

8. Deutsch AL, Coel MN, Mink JH. Imaging of stress injuries to bone. Radiography, scintigraphy, and MR imaging. Clin Sports Med 1997;16:275-90. https://doi.org/10.1016/S0278-5919(05)70022-3

9. Labovitz JM, Schweitzer ME. Occult osseous injuries after ankle sprains: incidence, location, pattern, and age. Foot Ankle Int 1998;19:661-7. https://doi.org/10.1177/107110079801901003

10. Saxena A, Eakin C. Articular talar injuries in athletes: results of microfracture and autogenous bone graft. Am J Sports Med 2007;35:1680-7. https://doi.org/10.1177/0363546507303561

11. Harmon KG. The ankle examination. Prim Care 2004;31:1025-37. https://doi.org/10.1016/j.pop.2004.07.008

12. Konradsen L, Voigt M, Højsgaard C. Ankle inversion injuries. The role of the dynamic defense mechanism. Am J Sports Med 1997;25:54-8. https://doi.org/10.1177/036354659702500110

13. Longo UG, Loppini M, Berton A, Marinozzi A, Maffulli N, Denaro V. The FIFA 11+ program is effective in preventing injuries in elite male basketball players: a cluster randomized controlled trial. Am J Sports Med 2012;40(5):996-1005. https://doi.org/10.1177/0363546512438761

14. Nishimura G, Yamato M, Togawa M. Trabecular trauma of the talus and medial malleolus concurrent with lateral collateral ligamentous injuries of the ankle: evaluation with MR imaging. Skeletal Radiol 1996;25:49-54. https://doi.org/10.1007/s002560050031 